\title{
Co-occurring WARS2 and CHRNA6 mutations in a child with a severe form of
} infantile parkinsonism

Running title: WARS2 and CHRNA6 variants in infantile parkinsonism

Simone Martinelli, PhD, ${ }^{1,16}$ Viviana Cordeddu, $\mathrm{PhD},{ }^{2,16}$ Serena Galosi, MD, $\mathrm{PhD},{ }^{3,16}$ Ambra Lanzo, PhD, ${ }^{4}$ Eleonora Palma, PhD, ${ }^{5,6}$ Luca Pannone, PhD, ${ }^{1,7}$ Andrea Ciolfi, PhD, ${ }^{7}$ Michela Di Nottia, PhD, ${ }^{8}$ Teresa Rizza, $\mathrm{PhD},{ }^{8}$ Gianfranco Bocchinfuso, $\mathrm{PhD},{ }^{9}$ Alice Traversa, PhD, ${ }^{10}$ Viviana Caputo, PhD, ${ }^{10}$ Andrea Farrotti, PhD, ${ }^{9}$ Claudia Carducci, PhD, ${ }^{10}$ Laura Bernardini, PhD, ${ }^{11}$ Susanna Cogo, PhD, ${ }^{12}$ Maria Paglione, $\mathrm{MS},{ }^{4}$ Martina Venditti, MS, ${ }^{3,7}$ Annarita Bentivoglio, ${ }^{13}$ Joanne Ng, PhD, ${ }^{14}$ Manju A. Kurian, MD, ${ }^{14}$ Laura Civiero, $\mathrm{PhD},{ }^{12}$ Elisa Greggio, PhD, ${ }^{12}$ Lorenzo Stella, $\mathrm{PhD},{ }^{9}$ Flavia Trettel, PhD, ${ }^{5}$ Miriam

Sciaccaluga, PhD, ${ }^{15}$ Cristina Roseti, $\mathrm{PhD},{ }^{6}$ Rosalba Carrozzo, MD, ${ }^{8}$ Sergio Fucile, $\mathrm{PhD}, 5,15$ Cristina Limatola, $\mathrm{PhD},{ }^{5,15}$ Elia Di Schiavi, $\mathrm{PhD},{ }^{4}$ Marco Tartaglia, $\mathrm{PhD},{ }^{7,17}$ Vincenzo Leuzzi, MD ${ }^{3,17}$

${ }^{1}$ Department of Oncology and Molecular Medicine, Istituto Superiore di Sanità, Rome, Italy. ${ }^{2}$ National Center for Drug Research and Evaluation, Istituto Superiore di Sanità, Rome, Italy. ${ }^{3}$ Department of Neuroscience, Sapienza University of Rome, Rome, Italy. ${ }^{4}$ Institute of Bioscience and BioResources, National Research Council, Naples, Italy. ${ }^{5}$ Department of Physiology and Pharmacology, Sapienza University of Rome, Rome, Italy. ${ }^{6}$ IRCCS San Raffaele Pisana, Rome, Italy. ${ }^{7}$ Genetics and Rare Diseases Research Division, Ospedale Pediatrico Bambino Gesù, IRCCS, Rome, Italy. ${ }^{8}$ Muscular and Neurodegenerative Disorders Unit, Ospedale Pediatrico Bambino Gesù, IRCCS, Rome, Italy. ${ }^{9}$ Department of Chemical Science and Technologies, Tor Vergata University of Rome, Rome, Italy. ${ }^{10}$ Department of Experimental Medicine, Sapienza University of Rome, 
Rome, Italy. ${ }^{11}$ Cytogenetics Unit, IRCSS Casa Sollievo della Sofferenza Foundation, San Giovanni Rotondo (FG), Italy. ${ }^{12}$ Department of Biology, University of Padua, Italy. ${ }^{13}$ Centro per i Disturbi del Movimento, Università Cattolica del Sacro Cuore, Rome, Italy ${ }^{14}$ Molecular Neurosciences, Developmental Neurosciences, UCL Institute of Child Health, London, UK. ${ }^{15}$ IRCCS Neuromed, Pozzilli (IS) Italy. ${ }^{16}$ These authors equally contributed to the manuscript. ${ }^{17}$ These authors jointly coordinated this work.

\section{Correspondence to:}

Dr. Marco Tartaglia, Genetics and Rare Diseases Research Division, Ospedale Pediatrico Bambino Gesù, IRCCS, Rome, Italy; email:marco.tartaglia@opbg.net; telephone number +3906 68593742; fax number +390668594584

or

Prof. Vincenzo Leuzzi, Department of Pediatrics and Child Neurology and Psychiatry, Sapienza University of Rome, Rome, Italy; email: vincenzo.leuzzi@uniroma1.it; telephone number +3906 49972912; fax number +39064440232

Number of characters in the title: 95

Number of characters in the running title: 51

Number of words in the Abstract: 232

Number of words in the main text: 1995

Number of figures: 2

Number of color figures: 2

Supplementary data: Supplementary Materials and Methods, Supplementary Results and Figures, Videos 1-8 and Videos' legends, Supplementary Tables 1 and 2 References: 12 
Key words: infantile parkinsonism, aminoacyl-tRNA synthetase, WARS2, nicotinic acetylcholine receptor, CHRNA6.

\section{Financial Disclosure/Conflicts of Interest}

The authors report no conflicts of interest relevant to the manuscript.

\section{Study funding}

This work was supported by grants from Fondazione Bambino Gesù (Vite Coraggiose, to MT), Italian Ministry of Health (Ricerca Corrente 2016, 2017 and 2018, to AC, MS, SF, CL and MT, and RF2009-1473235, to EDS), Italian Ministry of Instruction, University and Research (FIRB RBNE08LN4P_002 to EDS and PRIN 2008JKH2MM_002 to AL), AICEFIRE and UCB Pharma to EP, and the Michael J Fox Foundation for Parkinson Research (12080 to LC and EG). 


\section{Abstract}

Objective. To investigate the molecular cause(s) underlying a severe form of infantileonset parkinsonism and characterize functionally the identified variants.

Methods. A trio-based whole exome sequencing (WES) approach was used to identify the candidate variants underlying the disorder. In silico modeling, and in vitro and in vivo studies were performed to explore the impact of these variants on protein function and relevant cellular processes.

Results. WES analysis identified biallelic variants in WARS2, encoding the mitochondrial tryptophanyl tRNA synthetase (mtTrpRS), a gene whose mutations have recently been associated with multiple neurological phenotypes, including childhood-onset, levodoparesponsive or unresponsive parkinsonism in a few patients. A substantial reduction of mtTrpRS levels in mitochondria and reduced OXPHOS function was demonstrated, supporting their pathogenicity. Based on the infantile-onset and severity of the phenotype, additional variants were considered as possible genetic modifiers. Functional assessment of a selected panel of candidates pointed to a de novo missense mutation in CHRNA6, encoding the $a 6$ subunit of neuronal nicotinic receptors, which are involved in the cholinergic modulation of dopamine release in the striatum, as a second event likely contributing to the phenotype. In silico, in vitro (Xenopus oocytes and GH4C1 cells) and in vivo (C. elegans) analyses demonstrated the disruptive effects of the mutation on acetylcholine receptor structure and function.

Conclusion. Our findings consolidate the association between biallelic WARS2 mutations and movement disorders, and suggest CHRNA6 as a genetic modifier of the phenotype.

Glossary: ACh, acetylcholine; DA, dopaminergic; EOP, early-onset parkinsonism; HVA, homovanillic acid; mtARSs, mitochondrial aminoacyl-tRNA synthetases; mtTrpRS, mitochondrial tryptophanyl tRNA synthetase; MTS, mitochondrial targeting sequence; 
nAChR, nicotinic acetylcholine receptor; OXPHOS, oxidative phosphorylation; PD,

Parkinson's disease; RCCs, respiratory chain complexes; SEP, sensory evoked potentials; VEP, visual evoked potentials; WB, Western blot; WES, whole-exome sequencing. 


\section{Introduction}

Early-onset parkinsonism (EOP) is an exceedingly rare condition, usually of monogenic origin, caused by aberrant neurotransmitter synthesis, vesicular trafficking, autophagy, or mitochondrial function [1]. A significant proportion of subjects with a recognized primary genetic cause also shows concomitant variant(s) in Parkinson's disease (PD)-associated genes, acting as phenotypic modifiers which explain, in part, the wide variability of disease onset and progression [2].

Mitochondrial aminoacyl-tRNA synthetases (mtARSs) are essential components of the translation machinery of mitochondria, charging tRNAs with their cognate amino acids during translation of mitochondrial genes [3]. mtARS gene mutations have recently emerged as the molecular cause underlying a wide spectrum of human diseases. Among these, biallelic variants affecting WARS2, the gene encoding the tryptophanyl mtARSs (mtTrpRS), have been reported in subjects with a neurological presentation [4]. More recently, the phenotypic spectrum associated with WARS2 mutations has been expanded by the identification of two individuals with EOP $[5,6]$.

CHRNA6 encodes the a6 subunit of neuronal nicotinic receptors ( $\mathrm{nAChRs),} \mathrm{is} \mathrm{highly}$ expressed presinaptically in dopaminergic (DA) neurons of the nigrostriatal-mesolimbic pathway, is activated by acetylcholine/nicotine binding, and is involved in the cholinergic modulation of dopamine release in the striatum [7].

Here, we report co-occurrence of biallelic WARS2 mutations and a de novo CHRNA6 variant in a child with a severe form of infantile parkinsonism. Our data confirm the association between WARS2 loss-of-function and movement disorders, and suggest a role of CHRNA6 as a modifier gene in EOP.

\section{Methods}

Methods are reported as Supplementary Information. 


\section{Results}

Case report

The proband (AV07) is an 11 year-old male born from non-consanguineous, healthy parents. Between 6-9 months, trunk instability and occasional action jerks of limbs were noticed (video 1). At 10-15 months, loss of postural control, axial hypotonia, dysarthria, and dysphagia became evident. Social interaction and language comprehension remained relatively spared. At 15 months, CSF examination revealed low homovanillic acid (HVA, $185 \mathrm{nmol} / \mathrm{L}$; n.r.295-932), neopterin (7.8 nmol/L; n.r.12-30), and biopterin $(5.5 \mathrm{nmol} / \mathrm{L}$; n.r.15-40). Brain MRI, ${ }^{1} \mathrm{H}-\mathrm{MRS}$, EEG, flash and pattern VEP, and SEP were normal. Between 18-36 months, the child developed dystonic posturing of limbs, bradykinesia, rigidity and subcontinuous generalized rest and action rhythmic jerk-like movements (video 2). Levodopa-carbidopa treatment $(5 / 1.25 \mathrm{mg} / \mathrm{kg} / \mathrm{day})$ was started at the age of 2 years with relevant motor improvement (video 3), which progressively vanished at the age of 4 with the emergence of on/off phenomena and peak dose dyskinesia (video 4). The child also developed oculogyric crisis, ptosis, supranuclear gaze palsy, exotropia, hypomimia, a severe rigid-akinetic condition (videos 5,6), and versive seizures associated with paroxysmal epileptic alterations in frontal lobe on EEG recording. CSF examination revealed a further decrease of HVA (77 nmol/L; n.r.211-871). Mild cerebral atrophy was evident on brain MRI (Fig. 1A). At 6 years, DaTSCAN imaging revealed a severe derangement of DA striatal pathways (Fig. 1B). Worsening of on-off phenomena required progressive increased levodopa-carbidopa dosage (up to $10 / 2.5 \mathrm{mg} / \mathrm{kg} /$ day). Dopamine receptor agonists, COMT and MAO-B inhibitors were ineffective. At the age of 7 , levodopa-carbidopa intestinal gel (240 mg/day) resulted in a relative clinical stabilization for a few years, followed by a subcontinuous levodopa-induced dyskinetic status in waking (video 7). 


\section{Molecular findings}

A trio-based WES analysis allowed to exclude occurrence of mutations in known EOPassociated genes and hemizygous hits compatible with X-linked inheritance. Among biallelic events, WES identified compound heterozigosity for two missense variants, c.37T>G (p.Trp13Gly) and c.679A>G (p.Met227Val), in WARS2 (Fig. 2A, Supplementary Table 1). Both variants were predicted to be "deleterious" by CADD. p.Met227Val affected an invariant residue among WARS2 orthologs, and was not reported in public databases (gnomAD/ExAC), while p.Trp13Gly was reported in gnomAD (allele frequency=3.265e $\mathrm{e}^{-3}$ ). Furthermore, Sanger sequencing validated three de novo variants affecting genes not previously associated with human disease, but encoding proteins with relevant function in neurodevelopment/neurophysiology (CHRNA6, HIBADH, and PAK6). These variants were either private or rare $(<1 / 20,000$ in gnomAD), were predicted to be deleterious by CADD (Supplementary Table 1), and were also identified in buccal mucosal epithelial cells and skin fibroblasts, supporting their germline origin. Interrogation of GeneMatcher failed in identifying any relevant match, and mutation scanning performed in two small EOP cohorts was negative, excluding a major role of these genes in EOP.

\section{Functional studies}

WARS2 contains an $N$-terminal mitochondrial targeting sequence (MTS) and a $C$-terminal domain with Trp-tRNA ligase activity (Fig. $2 A) \cdot \operatorname{Trp}^{13}$ is located within the MTS, and its substitution into glycine was previously shown to cause impaired mitochondrial localization [4]. No functional information was available for p.Met227Val (ligase domain). To explore the effect of these lesions, WB analysis was performed in isolated organelles, demonstrating a substantial reduction of WARS2 levels in patient's fibroblasts compared to controls (Fig. 2B). Indirect evaluation of the oxidative phosphorylation (OXPHOS) status was assessed in mitochondria. Quantification of complex V activity showed a significant reduction of ATP synthesis using different substrates (16\%, succinate; $23 \%$, malate; $21 \%$, 
pyruvate/malate) (Fig. 2C), indicating impaired function of the respiratory chain complexes (RCCs), while no difference was detected in the expression of RCCs subunits (Fig. 2D). Functional validation of PAK6 and HIBADH variants ruled out their contribution to the phenotype (Supplementary Results, Supplementary Fig. 1). In contrast, the CHRNA6 c.527T>C (p.Phe176Ser) variant was shown to disrupt protein structure (Supplementary Results, Supplementary Fig. 2A,B) and receptor function. Specifically, different combinations of cDNA clones encoding human $\alpha 6, \beta 2, \beta 3$ and $\beta 4$ subunits were injected in Xenopus oocytes, and ACh-evoked currents were recorded by voltage-clamp technique. Oocytes expressing $\alpha 6 / \beta 4$ or $\alpha 6 / \beta 3 / \beta 4$ displayed functional receptors, while those expressing $\alpha 6^{\mathrm{F} 176 \mathrm{~S}} / \beta 4$ did not (Supplementary Table 2). Of note, oocytes expressing $\alpha 6^{\mathrm{F} 176 S / \beta 3 / \beta 4}$ showed strongly reduced current amplitudes and number of responsive cells (Supplementary Table 2, Supplementary Fig. 2C-E). Consistently, GH4C1 cells expressing $\alpha 6 / \beta 4$ displayed functional receptors, while those expressing $\alpha 6^{\mathrm{F} 176 S / \beta 4}$ did not, with expression of the mutant subunit causing a significant reduction in current density and number of responsive cells (Supplementary Fig. 2F-H). Collectively, these data demonstrated a loss-of-function role of p.Phe179Ser in Xenopus oocytes and GH4C1 expression systems.

To validate these findings in vivo, we used $C$. elegans as an experimental model. The assembly of the ACh receptor is well-conserved in nematodes. Among a subunits, unc-63 more closely resembles the human gene (https://www.ncbi.nlm.nih.gov/UniGene). In muscles, UNC-63 is part of the levamisole-sensitive receptor [(L)-AChR], which is susceptible to hypercontraction/paralysis induced by the nicotinic agonist levamisole [8]. UNC-63 is required for locomotion, with null mutants being uncoordinated and resistant to levamisole. Transgenic animals overexpressing unc-63F169S (homolog of hCHRNA6 F176S) under the control of pmyo-3, driving expression in body-wall muscles, showed normal locomotion in crawling and thrashing assays, excluding a dominant-negative effect of the 
mutation (Supplementary Fig. 3A,B). However, while overexpression of $u n c-63^{W T}$ in an unc-63-- background ( $x 37$ ) rescued both uncoordinated phenotype and levamisole resistance, expression of the mutant allele did not (Supplementary Fig. 3 C,D; video 8), demonstrating a loss-of-function role of p.Phe169Ser in muscle receptors. Since muscle and neuronal AChRs exhibit different pharmacological profiles $[9,10]$, we explored the functional impact of the variant in neurons by evaluating the sensitivity to the nicotinic agonist DMPP, whose toxicity is strictly controlled by neuronal UNC-63-containing nAChRs [11]. Exposure of wild-type animals to DMPP caused a lethal phenotype at the L2-to-L3 molt stage, while unc-63\% mutants exhibited partial resistance to the drug (Supplementary Fig. 3E). Neuronal expression of unc-63 ${ }^{W T}$ (punc-63::unc-63) in unc-63-1mutants partially rescued DMPP sensitivity, while expression of unc-63F169S did not. More importantly, wild-type worms expressing unc-63F169S in neurons acquired partial resistance to the drug, indicating a dominant-negative effect of the mutation in neuronal receptors.

Based on these findings, compassionate use of nicotine was considered as a therapeutic option, with only transient improvement of motor symptoms (Supplementary Results).

\section{Discussion}

We report on a severe form of infantile-onset parkinsonism, whose clinical course and response to therapy recapitulated adult PD. WES analysis and functional validation studies identified biallelic mutations in WARS2 as the molecular cause of the disease and a CHRNA6 variant potentially acting as a genetic modifier .

WARS2 is a nuclear-encoded protein fundamental to the mitochondrial synthesis of RCCs subunits. Biallelic WARS2 mutations had previously been reported in sixteen subjects with a phenotype ranging from mitochondrial encephalopathy to variably less severe conditions, including dystonia, epilepsy and ID [4-6]. More recently, WARS2 
mutations have been identified in two patients with childhood-onset parkinsonism $[5,6]$. Interestingly, in one of these subjects, mutations affected the same and the adjacent residues found to be mutated in the present case, suggesting possible genotype/phenotype correlation. Previous studies documented that p.Trp13Gly impacts proper subcellular localization [5] and variably affect the OXPHOS system [6], which are in line with the present findings. Compared to the reported EOP subjects $[5,6]$, however, our patient showed a more severe phenotype, with first signs of disease at 6 months, and rapidly progressive neurological deterioration. CSF examination disclosed reduced HVA, a biochemical marker not previously associated with WARS2 defects. Levodopa/carbidopa treatment was instituted at 24 months with a short "honeymoon" response, followed by increasingly severe motor fluctuations leading to the need for levodopa/carbidopa intestinal gel (7 years). DaTSCAN was confirmatory, revealing a complete derangement of the DA striatal pathways. Collectively, these findings suggest possible occurrence of a second event modulating the phenotype.

Among three de novo variants in candidate modifier genes, multiple lines of evidence pointed to the $\mathrm{c} .527 \mathrm{~T}>\mathrm{C}$ transition affecting CHRNA6. Structural modelling and functional profiling demonstrated a disrupting impact of this variant on protein structure and receptor function. Specifically, p.Phe176Ser exhibited a loss-of-function behavior in Xenopus oocytes, GH4C1 cells and $C$. elegans neuromuscular junction, while it displayed a dominant-negative effect in neuronal receptors of the nematode. $\alpha 6^{*} n A C h R s$ have been extensively studied to explore their potential role in PD. Data from preclinical models established that striatal $\mathrm{nAChRs}$ influence several biological processes relevant to motor function [12]. However, mice lacking $\alpha 6, \alpha 4$, or both subunits, show only minor motor deficits [7], possibly because of functional redundancy. Consistently, CHRNA6 is not an essential gene given the relatively high number of loss-of-function variants (gnomAD, pLI= 0). Our data do not support a role for CHRNA6 as a new EOP-associated gene; rather, 
they suggest a role of this gene as a modifier able to exacerbate the clinical presentation associated with biallelic WARS2 mutations (or any other EOP-associated lesion), thus contributing to the unique biochemical (low HVA) and imaging (DaTSCAN) profile of the disease. A transient improvement in motor symptoms and levodopa/carbidopa effectiveness observed after administration of nicotine at an early stage of the disease support this hypothesis, and suggest that nicotine may improve dopamine response in the early stage of PD, in line with data indicating a neuroprotective role of nicotine against nigrostriatal DA neuronal loss [12].

In summary, our findings extend the association between biallelic WARS2 mutations and EOP, and suggest CHRNA6 as a genetic modifier of the phenotype. In line with accumulating evidence indicating the relevance of multilocus variation in explaining phenotypic variability, our data emphasize the importance of functional profiling to appreciate the relative contribution of genomic variants to the clinical phenotype.

\section{Figure Legends}

Figure 1. Brain imaging. (A) Brain MRI of AV07 (5 years) showed mild cerebral atrophy and mega cisterna magna. (B) Brain imaging with single-photon emission CT DaTSCAN (6 years) revealed a severe derangement of dopaminergic striatal pathways (left), compared to an age- and sex-matched healthy control (right).

Figure 2. Functional impact of WARS2 mutations. (A) Location of disease-causing mutations is reported above the WARS2 domain structure scheme (lesions identified in EOP are shown in blue). Mutations found in the present study (red) are reported below the cartoon. The mitochondrial targeting sequence (MTS) and the aminoacyl-tRNA synthetase conserved site are shown in red and yellow, respectively. (B) A significant reduction of WARS2 levels was documented in mitochondria from patients' fibroblasts compared to 
control cells ( ${ }^{*} P<0.05 ;{ }^{* *} P<0.0005$; Student's t test). WARS2 levels were normalized against VDAC. (C) Spectrophotometric determination of complex V activity. Significantly reduced ATP synthesis was documented in mitochondria of patient's fibroblasts, with either substrates used (succinate, malate, pyruvate/malate) $(P<0.05)$. Data are expressed as mean $\pm S D$ of three independent experiments. (D) Expression of individual subunits of the mitochondrial CI (NDUFS1, NDUFA9), CII (SDHA), CIII (UQCRC2), CIV (COXII), and CV (ATP5A1) complexes. 


\section{Declarations of interest}

None.

\section{Funding}

This work was supported by grants from Fondazione Bambino Gesù (Vite Coraggiose, to MT), Italian Ministry of Health (Ricerca Corrente 2016, 2017 and 2018, to AC, MS, SF, CL and MT, and RF2009-1473235, to EDS), Italian Ministry of Instruction, University and Research (FIRB RBNE08LN4P_002 to EDS and PRIN 2008JKH2MM_002 to AL), AICEFIRE and UCB Pharma to EP, and the Michael J Fox Foundation for Parkinson Research (12080 to LC and EG).

\section{Final disclosures}

Ambra Lanzo was employed with Netcom Group S.p.A, Naples, Italy and founded by the Italian Ministry of Instruction, University and Research (PRIN 2008JKH2MM_002); Andrea Ciolfi was founded by the Italian Ministry of Health (Ricerca Corrente 2018 and 2019); Lorenzo Stella and Gianfranco Bocchinfuso were founded by the Italian Association for Cancer Research, by the Italian Ministry of University and Research and by the University of Rome Tor Vergata; Elia Di Schiavi was granted by the Telethon foundation (Full Project GGP16203), European Community (H2020-FETOPEN-1-2016-2017 801338) and CNR (Grant Joint Bilateral Agreement CNR/RFBR from CNR); Vincenzo Leuzzi has served on scientific advisory boards for Nutricia and BioMarin.

\section{Ethical approval and informed consent}

All authors declare that the manuscript is in accordance with the statement of ethical standards for manuscripts submitted to Parkinsonism and related disorders. Parents have 
consented for video publication and provided a signed release form authorizing the offline and/or online distribution of this video material.

\section{Data Availability}

Any anonymized data not published within the article will be shared by request from any qualified investigator.

\section{Authorship}

SM: conception and design of the study, analysis and interpretation of data, drafting the article, revising the manuscript critically for important intellectual content VC: conception and design of the study, analysis and interpretation of data, drafting the article, revising the manuscript critically for important intellectual content SG: conception and design of the study, analysis and interpretation of data, drafting the article, revising the manuscript critically for important intellectual content

$\mathrm{AL}$ : analysis and interpretation of data

EP: acquisition of data, analysis and interpretation of data

LP: analysis and interpretation of data

AC: analysis and interpretation of data

MDN: analysis and interpretation of data

TR: acquisition of data

GB: acquisition of data, analysis and interpretation of data

AT: acquisition of data

VC: acquisition of data

AF: acquisition of data

CC: acquisition of data 
LB: acquisition of data

SC: acquisition of data

MP: acquisition of data

MV: acquisition of data

AB: revising the manuscript critically for important intellectual content

$\mathrm{JN}$ : analysis and interpretation of data

MK: analysis and interpretation of data, revising the manuscript critically for important intellectual content

LC: acquisition of data, analysis and interpretation of data, revising the manuscript critically for important intellectual content

EG: acquisition of data, analysis and interpretation of data, revising the manuscript critically for important intellectual content

LS: acquisition of data, analysis and interpretation of data

FT: acquisition of data

MS: acquisition of data

CS: acquisition of data

$\mathrm{RC}$ : acquisition of data, analysis and interpretation of data, revising the manuscript critically for important intellectual content

SF: acquisition of data, analysis and interpretation of data, revising the manuscript critically for important intellectual content

CL: acquisition of data, analysis and interpretation of data, revising the manuscript critically for important intellectual content

EDS: acquisition of data, analysis and interpretation of data, revising the manuscript critically for important intellectual content MT: analysis and interpretation of data, drafting the article, revising the manuscript critically for important intellectual content 
VL: analysis and interpretation of data, drafting the article, revising the manuscript critically for important intellectual content

\section{Acknowledgements}

We thank Jean-Louis Bessereau (University of Lyon, Villeurbanne, France), Cori Bargmann (The Rockefeller University, New York, NY) and Peter Verhasselt (Jansen Research Foundation, Beerse, Belgium) for providing vectors used in $C$. elegans and Xenopus studies. We are grateful to Serenella Venanzi (Istituto Superiore di Sanità, Rome, Italy), Giuseppina Zampi (Institute of Biosciences and BioResources, CNR, Naples, Italy) and Francesco Pennica for their technical assistance. C. elegans strains were provided by the Caenorhabditis Genetics Center, which is funded by NIH Office of Research Infrastructure Programs (P40 OD010440). We also thank WormBase, Dr. Wortmann (Salzburg, Austria) for providing us the polyclonal rabbit anti-WARS2 antibody, and Paolo Bazzicalupo for his critical reading of the manuscript.

\section{References}

[1] A. Abeliovich, A.D. Gitler, Defects in trafficking bridge Parkinson's disease pathology and genetics, Nature 539 (2016) 207-216.

[2] S.J. Lubbe, V. Escott-Price, J.R. Gibbs, M.A. Nalls, J. Bras, T.R. Price, A. Nicolas, I.E. Jansen, K.Y. Mok, A.M. Pittman, J.E. Tomkins, P.A. Lewis, A.J. Noyce, S. Lesage, M. Sharma, E.R. Schiff, A.P. Levine, A. Brice, T. Gasser, J. Hardy, P. Heutink, N.W. Wood, A.B. Singleton, N.M. Williams, H.R. Morris, Additional rare variant analysis in Parkinson's disease cases with and without known pathogenic mutations: evidence for oligogenic inheritance, Hum Mol Genet. 25 (2016) 5483-5489.

[3] M. Ibba, D. Soll, Aminoacyl-tRNA synthesis, Annu. Rev. Biochem 69 (2000) 69:617650. 
[4] L. Musante, K. Püttmann L, K. Kahrizi, M. Garshasbi, H. Hu, H. Stehr, B. Lipkowitz, S. Otto, L.R. Jensen, A. Tzaschach, P. Jamali, T. Wienker, H. Najmabadi, H.H. Ropers, A.W. Kuss, Mutations of the aminoacyl-tRNA-synthetases SARS and WARS2 are implicated in the etiology of autosomal recessive intellectual disability. Hum Mutat. 38 (2017) 621-636. [5] E.A. Burke, S.J. Frucht, K. Thompson, L.A. Wolfe, T. Yokoyama, M. Bertoni, Y. Huang, M. Sincan, D.R. Adams, R.W. Taylor, W.A. Gahl, C. Toro, M.C.V. Malicdan, Biallelic mutations in mitochondrial tryptophanyl-tRNA synthetase cause Levodopa-responsive infantile-onset Parkinsonism, Clin Genet. 93 (2018) 712-718.

[6] M. Virdee, E. Swarnalingam, M. Kozenko, M. Tarnopolsky, K. Jones, Expanding the Phenotype: Neurodevelopmental Disorder, Mitochondrial, With Abnormal Movements and Lactic Acidosis, With or Without Seizures (NEMMLAS) due to WARS2 Biallelic Variants, Encoding Mitochondrial Tryptophanyl-tRNA Synthase, J Child Neurol 2019: 0883073819854604.

[7] N. Champtiaux, J.P. Changeux, Knockout and knockin mice to investigate the role of nicotinic receptors in the central nervous system, Prog Brain Res 145 (2004) 235-251. [8] E. Culetto, H.A. Baylis, J.E. Richmond, H.A. Baylis, J.E. RichmonD, A.K. Jones, J.T. Fleming, M. D. Squire, J.A. Lewis, D.B. Sattelle, The Caenorhabditis elegans unc-63 gene encodes a levamisole-sensitive nicotinic acetylcholine receptor alpha subunit. J Biol Chem. 279 (2004) 42476-42483.

[9] T. Boulin, M. Gielen, J.E. Richmond, D.C. Williams, P. Paoletti, J.L. Bessereau, Eight genes are required for functional reconstitution of the Caenorhabditis elegans levamisolesensitive acetylcholine receptor, Proc Natl Acad Sci U S A 105 (2008) 18590-18595. [10] M. Jospin, Y.B. Qi, T.M. Stawicki, T. Boulin, K.R. Schuske, H.R.Horvitz, J.L. Bessereau, E.M. Jorgensen, Y. Jin, A neuronal acetylcholine receptor regulates the balance of muscle excitation and inhibition in Caenorhabditis elegans, PLoS Biol. 7 (2009) e1000265; 1-17. 
[11] A.F. Ruaud, J.L. Bessereau, Activation of nicotinic receptors uncouples a

developmental timer from the molting timer in C. elegans, Development 133 (2006) 22112222.

[12] M. Quik, K. O'Leary, C.M. Tanner. Nicotine and Parkinson's disease: implications for therapy. Mov Disord. 23(2008) 1641-1652. 